\title{
Study on Steering by Wire Controller Based on Improved $\mathrm{H} \infty$ Algorithm
}

\author{
http://dx.doi.org/10.3991/ijoe.v9iS2.2569 \\ Huipeng Chen ${ }^{1,2}$, Lingjun Zhu ${ }^{1}$, Xiaofan Liu ${ }^{1}$, Sunan $\mathrm{Yu}^{1}$, and Dingcheng Zhao ${ }^{1}$ \\ 1 School of Mechanical Engineering, HangZhou DianZi University, Hangzhou, China \\ ${ }^{2}$ Postdoc of Huazhong University of Science \& Technology, Wuhan, China
}

\begin{abstract}
With the continuous development of vehicle and electronic industry, SBW (Steering by Wire) is replacing the traditional steering device of vehicle. Firstly this paper introduces the principle and structure of SBW, then discusses how to model the kinetic equation of SBW and how to simplify the model reasonably, and then obtains the control model of SBW. To meet the system requirement of robust performance, this paper selects the control strategy based on $\mathrm{H} \infty$ algorithm. According to the further observation and study on the curve of $S / T$ singular value in $\mathrm{H} \infty$ algorithm, put forward a method which constructs a closed loop function and then back-step the system controller on the basis of key parameter in system frequency. The analysis of the time domain and the frequency domain of SBW, the simulation result under the interference and parameter perturbation of the model show that the controller designed by this method is simple and effective, and the controller owns perfect robust stability and robust performance.
\end{abstract}

Index Terms - Steering by Wire, Robust, $\mathrm{H} \infty$ algorithm.

\section{INTRODUCTION}

With the continuous development of electronic and control technology recently, electronic power steering of the vehicle has a rapid development. The application of the various kinds of active and power steering system improves the transfer characteristic of steering angle, the steering response characteristic and the stability of steering for vehicle $[1,2]$. However, nowadays the mechanical connect of the steering system leads that the steering performance has a strong non-linear timevarying characteristic with the change of speed, steering angle and the condition of the road adhesion. To make the vehicle drive along the expected path, drivers must adjust the characteristics of themselves frequently, so it would increase the mental and physical burden, and the nonprofessional driver would not adapt it especially [3]. So many researchers from different countries are studying the new technology for the steering system to solve the above problems, under this circumstance, the Steering-By-Wire system (SBW) emerge as the times requires. In recent decade, almost all the large scale automobile manufacturers have carry out deep studies on SBW. And the new technology has been applied in many new automobiles, such as the concept vehicle C-Grosser of CITROEN Corporation in France, the concept vehicle 8129 of the Daimler Chrysler Corporation in Germany, the concept vehicle Ray of KIA Corporation in South Korea and the test vehicle FASCarll designed by the
German aerospace centre and traffic technology study institute in 2011. What's more, the manufacturers of the components, the vehicle design corporations and many universities have studied the SBW.

The main object of studying SBW control strategy is how to keep the stability, tracking and anti-interference under the complicated condition of work and road. At present, there are a large number of control strategies used in the SBW, and several typical cases acquire some good effect, for instance PID, LQG, Hळ etc [4, 5]. Aiming at the unreasonableness of design object and interference limit of LQG, the scholar Zames from Canada put forward the control idea of $\mathrm{H} \infty$ [6]. After more than twenty years development, $\mathrm{H} \infty$ control theory has been one of theoretical systems that solve the robust control problems successfully and perfectly. The selection for the weighting function is crucial and it will determine the performance of SBW. However, to select a appropriate weighting function should rely on the good engineer experience of designing SBW, and should take iterative and a lot of calculus, these problems bring many difficulties in designing the $\mathrm{H} \infty$ controller of SBW.

This paper avoids selecting the weight function with the method of reverse which is based on improving the $\mathrm{H} \infty$ control algorithm. This method constructs the closed-loop transfer function of system based on the $\mathrm{H} \infty$ algorithm directly with the further observation and study on the S/T singular curve by the author, and then reserves a closed-loop system with an expected $\mathrm{S} / \mathrm{T}$ curve which meets the robust performance requirements of SBW. This is a kind of simplified $\mathrm{H} \infty$ circuit shaping algorithm based on the engineer significance. Its physical conception is distinct and the solving process is very easy, the orders of final controller are low. The S/T curve and the result of the simulation on the step response show that the robust stability and the robust performance of improved $\mathrm{H} \infty$ algorithm are better than the traditional $\mathrm{H} \infty$ algorithm.

\section{Kinetic Model Of SBW AND ItS ANALYSIS}

SBW adopts the X-By-Wire technology (XBW), and transfers the signal to the Electronic Control Unit (ECU), and then the commands transmitted by ECU will control the steering execute assembly to finish the steering commands, and finally the driver realize the driving intention. SBW gets rid of the limit of traditional mechanical connect completely. It can design transfer characteristics of the angle and force freely in theory and it offers tremendous space to design the steering 
characteristics. It has a extensive application market and vast development potential.

\section{A. Theory of SBW Structure}

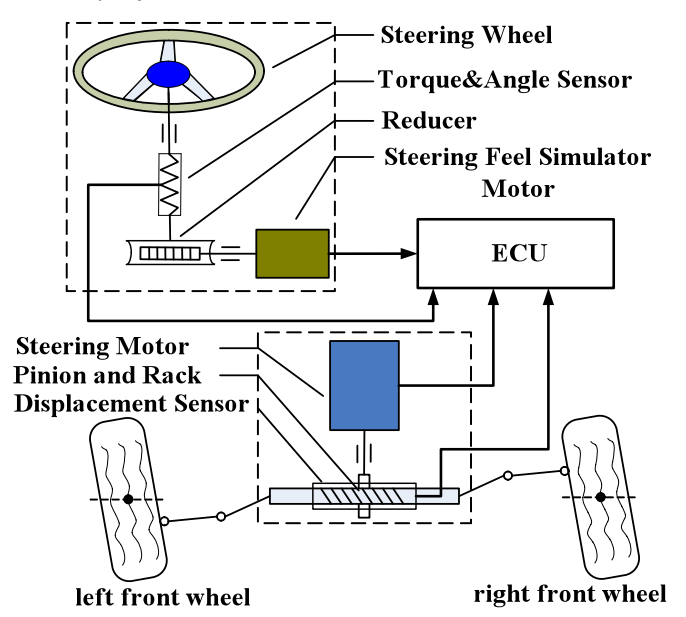

Figure 1. Theory schematic diagram of SBW structure

The structure and theory schematic diagram of SBW studied in this paper is shown in figure 1, mechanical part contains the hand wheel part and the steering wheel part. The hand wheel part is used to simulate the transfer of driver's intention and the torque of the hand wheel; it contains the following main hardware: motor for simulating the steering feel, decelerating mechanism, rotation sensor, current sensor, torque sensor, general hand wheel and steering column. Steering part's function is to make the steering wheel turn; it contains the motor for executing turning, decelerating mechanism, rotation sensor, current sensor, rack displacement sensor, general steering gear-rack. Apart from the mechanical hardware, the distinct difference between SBW and traditional steering system is that $\mathrm{SBW}$ is equipped with a controller which has three functions: control the motor for simulating the steering feel, control the motor for executing turning and the fault tolerant control for the whole system's main components.

\section{B. Kinetic Model of $S B W$}

As the figure 2 shown, SBW is divided into two parts according to the motor position, and the figure 2(a) shows the schematic diagram force of the simulation steering feeling assembly and the hand wheel assembly, and figure 2(b) shows the schematic diagram force of executing turning assembly.

In figure 2(a), $I_{h}, D_{h}$ and $D_{m}$ represent the moment of inertia of the hand wheel, damping coefficient of steering column and motor of simulating steering feel respectively. $T_{h}$ is the steering torque and $T_{h r}$ is resistance torque of the hand wheel. $T_{t s}$ is resistance torque measured by torque sensor. $T_{m}$ and $T_{m r}$ represent output torque and resistance torque of motor. $\delta_{h}$ and $\delta_{m}$ represent the angle of the hand wheel and the motor of simulating steering feel. $f\left(\delta_{h}, \dot{\delta}_{h}\right), f\left(\delta_{m}, \dot{\delta}_{m}\right)$ represent the nonlinear dry friction resistance torque of the hand wheel assembly and the motor for simulating the steering feel. and its kinetic equation as following:

$$
\begin{aligned}
& I_{h} \ddot{\delta}_{h}+D_{h} \dot{\delta}_{h}+f\left(\delta_{h}, \dot{\delta}_{h}\right)=T_{h}-T_{h r} \\
& I_{m} \ddot{\delta}_{m}+D_{m} \dot{\delta}_{m}+f\left(\delta_{m}, \dot{\delta}_{m}\right)=T_{m}-T_{m r} \\
& T_{t s}=k_{t s}\left(\delta_{h}-i_{f w} x_{r}\right)+D_{t s}\left(\dot{\delta}_{h}-i_{f} \dot{x}\right)
\end{aligned}
$$

In figure 2(b), $I_{f}, D_{f}$ is the moment of inertia of the execute motor, damping coefficient, and its angle is $\delta_{m}$, the output torque is $T_{f}$ and the resistance torque is $T_{f r}$. $f\left(\delta_{f}, \dot{\delta}_{f}\right)$ is the resistance of nonlinear dry friction of motor for executing steering. And its kinetic equation as following:

$$
I_{f} \ddot{\delta_{f}}+D_{f} \dot{\delta}_{f}+k_{f} \delta_{f}+f\left(\delta_{f}, \dot{\delta}_{f}\right)=T_{f}-T_{f r}
$$

The model of small gear-rack is similar to the traditional steering system and the model can be expressed as following:

$$
m_{r} \ddot{x}_{r}+b_{r} \dot{x}_{r}+f\left(x_{r}, \dot{x}_{r}\right)=T_{r} / r_{p}-F_{r}+F_{d}
$$

$m_{r}$ and $b_{r}$ represents the quality of rack and its damping coefficient, $x_{r}$ is the displacement of rack. $T_{r}$ is the reaction torque which acts on the small gear-rack. $F_{r}$ is steering resistance and $F_{d}$ is random interference resistance. $i_{f w}$ is deceleration ratio of the motor for executing steering.

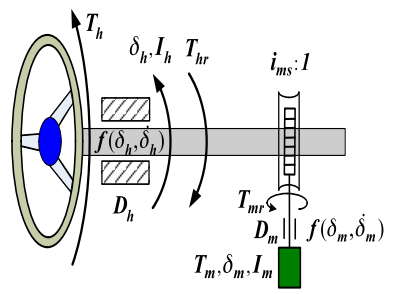

(a)

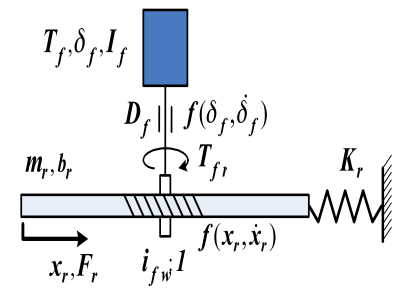

(b)
Figure 2. Force schematic diagram of SBW

According to the structure and force condition of SBW, the following relation can be concluded:

$$
T_{m r}=T_{h r} / i_{m s}, T_{w}=i_{f w} T_{f}, T_{h r}=T_{t s}, T_{r}=\rho T_{t s}
$$

In the above relation, $\rho$ is the coefficient of steering and $i_{m s}$ is the deceleration ratio of the motor for simulating steering feel. Based on the above equation from (1)-(5) and the middle relation, the following kinetic equation of SBW could be obtaining:

$$
\begin{aligned}
& i_{f w}\left(D_{t s} \dot{x}_{r}+k_{t s} x_{r}\right)+T_{h}= \\
& I_{h} \ddot{\delta}_{h}+\left(D_{h}+D_{t s}\right) \dot{\delta}_{h}+k_{t s} \delta_{h}+f\left(\delta_{h}, \dot{\delta}_{h}\right)
\end{aligned}
$$




$$
\begin{aligned}
& r_{p} m_{r} \ddot{x}_{r}+\left(r_{p} b_{r}+\rho_{0} D_{t s} i_{f w}\right) \dot{x}_{r}+\rho_{0} k_{t s} \dot{i}_{f w}{ }_{r}+f(x, \dot{x}) \\
& =\rho_{0}\left(D_{t s} \dot{\delta}_{h}+k_{t s} \delta_{h}\right)-F_{r}+r_{p} F_{d}
\end{aligned}
$$

\section{Simplify The Model and Analysis}

The resource of the steering resistance mainly comes from the effect between the road and tires, the inner steering system friction. The effect between the tire and the road is very complicated with the influence of the tire material, structure, pressure, vertical load and the working condition, and the inner friction of the system is quite complex, so the above factors lead that the steering resistance has the obvious nonlinear characteristic. In this paper, the overall analyze and accuracy model is not necessary for the steering resistance of the steering system. What we need is to grasp the relation between the front angle and the road resistance torque. So we can suppose the hypothesis that the relation between the front angle and road resistance torque is linear is tenable when we model based on the SBW.

Equivalent the aligning torque of front wheel as the linear spring and its stiffness is $K_{r}$, and it has the following expression:

$$
F_{r}=K_{r} x_{r}
$$

$f\left(x_{r}, \dot{x}_{r}\right)$ is the nonlinear dry friction torque of SBW, and its value is always small, so we can neglect it. So, the kinetic equation of SBW after simplifying:

$$
\begin{aligned}
& T_{h}+k_{s} i_{f w} x_{r}=I_{h} \ddot{\delta}_{h}+D_{h} \dot{\delta}_{h}+k \delta_{h} \\
& r_{p} m_{r} \ddot{x}_{r}+r_{p} b_{r} \dot{x}_{r}+\left(\rho_{0} k_{t s} i_{f w}+r_{p} K_{r}\right) x_{r}=\rho_{0} k \delta_{h}
\end{aligned}
$$

We can get the following relation via Laplace transform and arrange them:

$$
\begin{gathered}
X_{r}(s)=\rho k_{t s} \delta_{h}(s) / P(s), \\
X_{r}(s)=\rho k_{s} T_{h}(s) / Q(s), \\
\delta_{h}(s)=P(s) T_{h}(s) / Q(s)
\end{gathered}
$$

$X_{r}(s), \delta_{h}(s), T_{h}(s)$ is the Laplace transformation of $x_{r}, \delta_{h}, T_{h}$, and the expressions of $P(s), Q(s)$ as following:

$$
\begin{gathered}
Q(s)=u_{4} s^{4}+u_{3} s^{3}+u_{2} s^{2}+u_{1} s+u_{0}, \\
P(s)=r_{p} m_{r} s^{2}+r_{p} b_{r} s+u_{5}, u_{0}=k_{s} r_{p} b_{r}, \\
u_{1}=D_{h}\left(\rho k_{s} i_{f w}+r_{p} K_{r}\right)+k_{s} r_{p} K_{r}, \\
u_{2}=k_{s} r_{p} m_{r}+D_{h} r_{p} b_{r}+I_{h} u_{5}, u_{3}=r_{p}\left(b_{r} I_{h}+m_{r} D_{h}\right), \\
u_{4}=r_{p} m_{r} I_{h}, u_{5}=\rho k_{s} i_{f w}+r_{p} K_{r}
\end{gathered}
$$

\section{AnAlysis AND DeSign Of Robust CONTROL SYSTEM}

\section{A. Modeling The Control System of Sbw}

According to the analysis of the kinetic model, we can conclude the following system block diagram as shown in figure 3.

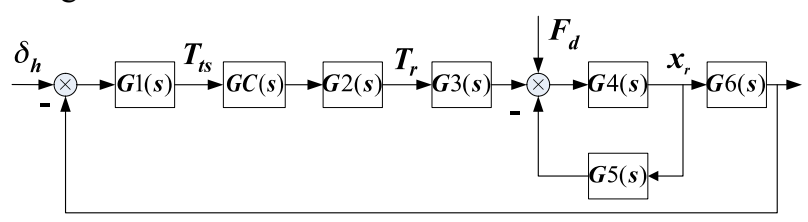

Figure 3. System block diagram of SBW

$$
G 1(s)=k_{t s}, G 2(s)=\rho, G 3(s)=1 / r_{p}, G 6(s)=i_{f w},
$$

$G 4(s)=1 /\left(m_{r} s^{2}+b_{r} s\right), G 5(s)=K_{r}$. Based on the work theory in the first chapter, SBW is an angle serve system actually that the controller control the power motor to offer the power according to the difference angle between the input and output that is the torque sensor signal. However, the road and work condition will affect the output signal from the sensor, and there is noise in the torque sensor, so the torque signal should be adjusted before transferring into the motor controller to make sure that the signal can be reflect the steering intention from the driver and then the power motor could be offer the accuracy power. Suppose the adjust controller $C(s)$, the improved mixed sensitivity $\mathrm{H} \infty$ circuit shaping in the robust control theory is used to design a controller which owns the robust stability and robust performance. Firstly, we supposed the input hand wheel angle $\delta_{h}=0$, and transform the original system block diagram into standard $\mathrm{H} \infty$ feedback structure as shown in figure 4. In the figure $4, T_{d}$ is the random interference from the road, $\Delta$ is the model perturbation which contains uncertain parameters and non-structural uncertainty. $W S, W R, W T$ is three extra weighting function to inhibit interference, limit control variable and improve the performance. $w$ is the virtual input to meet the standard control structure. And the controller of this whole system is $K(s)=G 2(s) G 3(s) G C(s)$, and the control object is $G(s)=G 1(s) G 4(s) G 6(s) /(1+G 4(s) G 5(s))$.

According to the SBW simulation parameters: $\rho=4$, $k_{t s}=121 \mathrm{Nm} / \mathrm{rad}, r_{p}=0.0088 \mathrm{~m}, i_{f w}=20, m_{r}=5.28 \mathrm{~kg}$, $b_{r}=326.6 \mathrm{~N} /(\mathrm{m} / \mathrm{s}), K_{r}=39951.6 \mathrm{~N} / \mathrm{m}$, we can obtain the specific transfer function expression:

$$
\begin{aligned}
& G 1(s)=121, G 2(s)=4, G 4(s)=1 /\left(5.28 s^{2}+326.6 s\right) \\
& , G 3(s)=1 / 0.0088, G 5(s)=39951.6, G 6(s)=20 . \\
& \text { So, we can finally calculate the control object } \\
& G(s)=2420 /\left(5.28 s^{2}+326.6 s+39951.6\right) \text {. }
\end{aligned}
$$

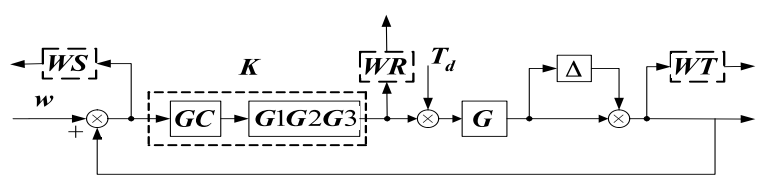

Figure 4. 4 Block diagram of controller 


\section{B. Improved Ho Robust Control Algorithm}

$\mathrm{H} \infty$ robust control is a theory that we can obtain a controller with the robust performance by optimizing the infinite norms of some performance index in the $\mathrm{H} \infty$ space (that is Hardy space, the following brief write down for $\mathrm{H})^{7}$. The $\mathrm{H} \infty$ robust control theory provides methods to solve the system robust control problems, for example the model may have uncertainties in a certain range and exist outside interference signal.

Uncertainty model includes two parts that one is uncertainty of non-structural uncertainty namely the dynamic characteristics of high frequency without modeling, another one is uncertainties of model parameters. The general means of modeling the uncertainty object uses an assembly to represent the object model; this assembly could be structured or unstructured. In this paper, the following unstructured multiplicative uncertainty is the unified expression of the above two uncertainty:

$$
G^{\prime}(s)=G(s)(1+\Delta)
$$

Generally speaking, multiplicative perturbation $\Delta$ has the high pass characteristic. What's more, we don't demand the display expression about $\Delta$ based on the standard frame of $\mathrm{H} \infty$, what we need is the knowledge of corresponding limit value $\left\|\Delta_{\max }\right\|_{\infty}$. Hळ mixed sensitivity control strategy shapes the closed transfer function such as sensitivity function and complementary sensitivity by gain shaping algorithm directly, so we can eliminate high peak value problem which may appear in the open loop gain shaping. Replace the effects of $\Delta$ with weighting function, and ensure the weighting function $W T(s)$ through $\left\|\Delta_{\max }\right\|_{\infty}$, and they offer great flexibility for the optimizing problem. Here we take the upper limit of $\Delta$ as the weighting function, and guarantee the system's robust stability with $W T(s)$ when the model is under perturbation, then can get rid of the $\Delta$ which is expressed in the original perturbation model, which means $\Delta=0$. Moreover the abilities to antiinterference and signal tracking can be guaranteed through weighting function $W S(s)$. But it is a key to choose the weighting function in this process; it determines the performance of control system directly. And to obtain the expected weighting function, designers should perform large calculus in repeated iteration to accumulate the practical experience, and there is no shortcut $^{8}$.

Based on the observe and study the curve of S/T mixed sensitivity singular value in the $\mathrm{H} \infty$ circuit shaping control, from the practical engineer significance, constructing the complementary sensitivity function $\mathrm{T}$ according to the bandwidth frequency, high frequency asymptote slope, the largest singular value and then back stepping the controller K. Ascertain the shape of sensitivity function $\mathrm{S}$ through the correlation of $\mathrm{S}$ and $\mathrm{T}$ indirectly, and then guarantee the robust stability and robust performance of system .

Suppose that the bandwidth frequency closed loop demanded is $1 / T_{1}$, to make the construction of $T$ easier, here the corner frequency is approximate to bandwidth, the frequency of high frequency asymptote is $-20 \mathrm{ndB}, \mathrm{n}$ is an integer which range from $1 \sim 3$, when $n$ is too large and the control order will rise, this phenomenon is adverse to the controller and the control effect is not improved obviously. To guarantee the system to track the target value with non static difference, choose the largest singular value equals to 1 and then construct complementary sensitivity function $\mathrm{T}$ as following:

$$
T=1 /\left(T_{1} s+1\right)^{n}=G K /(1+G K)
$$

The controller with the ideal S/T curve:

$$
K=1 /\left[\left(T_{1} s+1\right)^{n}-1\right] G
$$

The solving process is very easy which avoids a lot of iterative calculus on weighting function, and it is a simplified $\mathrm{H} \infty$ circuit shaping algorithm based on the engineer significance.

\section{Design The Control System of SBW}

To guarantee system to track the reference signal $\mathrm{w}$ with non static difference, choose the largest singular value of complementary sensitivity function equals to 1 . The bandwidth of system decides the respond speed and the SBW system demands great quality respond speed, so the value of bandwidth here is not less than $100 \mathrm{rad} / \mathrm{s}$. To restrain influence on the control error produced by the uncertain signal such as the noise of sensor, and guarantee the system's robust performance, choose the slope of high frequency asymptote equals to $-60 \mathrm{~dB} / \mathrm{dec}$. Thus the three order inertial system spectrum curve with the largest singular value 1 is constructed by the singular value curve of $\mathrm{T}$. To make the calculation convenient, the corner frequency is approximate to the bandwidth frequency, and then we obtain:

$$
\frac{1}{((1 / 100) s+1)^{3}}=\frac{G(s) K(s)}{1+G(s) K(s)}
$$

That is the controller of SBW:

$$
K(s)=\frac{5.28 s^{2}+326.6 s+39951.6}{0.00242 s^{3}+0.726 s^{2}+72.6 s}
$$

And then regulate the controller as following

$$
G C(s)=\frac{K(s)}{G 2(s) G 3(s)}=\frac{5.28 s^{2}+326.6 s+39951.6}{1.1 s^{3}+330 s^{2}+33000 s}
$$

To compare with the ordinary $H \infty$ mixed sensitivity control strategy, referring to the method to choose weighting function in the literature ${ }^{[6]}$, the this paper choose the three parameters of $\mathrm{H} \infty$ mixed sensitivity: $W S=15 /(s+0.5) \quad, \quad W R=0.01$

$W T=58(s+30) /(s+6000)$. By working out the function mixsyn(G,WS,WR,WT) with the aid of mixed sensitivity problem of the new robust control toolbox in Matlab, the process of designing the $\mathrm{H} \infty$ mixed 
sensitivity controller for the object $G(s)$ becomes more easier. And then we can conclude as following:

$$
K^{\prime}(s)=\frac{657039.8671(s+6000)\left(s^{2}+61.86 s+7567\right)}{(\mathrm{s}+0.5)(\mathrm{s}+481.2)(\mathrm{s}+5984)(\mathrm{s}+1.404 \mathrm{e} 004)}
$$

And then regulate the controller as following:

$$
\begin{aligned}
C^{\prime}(s) & =\frac{K^{\prime}(s)}{G 2(s) G 3(s)} \\
& =\frac{1445.4877(s+6000)\left(s^{2}+75 s+3616\right)}{(s+561.1)(s+1618)(s+5980)(s+0.5)}
\end{aligned}
$$

\section{Simulation AND AnAlysis On The CONTROL SYSTEM}

The controller designed by the improved $\mathrm{H} \infty$ algorithm is a three order controller, and the traditional $\mathrm{H} \infty$ controller designed by the choosing the weighting function is a four order controller.

By simulating and analyzing the unit step response of the rack displacement under the influence of two kinds controller and without controller, we can get the unit step response curve of front wheel angle as shown in figure 5 . According the curve, when without controller works, the settling time for the unit step response is 0.138 seconds and the overshoot is $63.8 \%$; when the improved $\mathrm{H}_{\infty}$ controller works, the settling time is 0.075 seconds and the settling time is 0.082 seconds when the traditional $\mathrm{H} \infty$ mixed sensitivity controller works. This shows that the improved $\mathrm{H} \infty$ controller has the better response performance when we guarantee the robust stability.

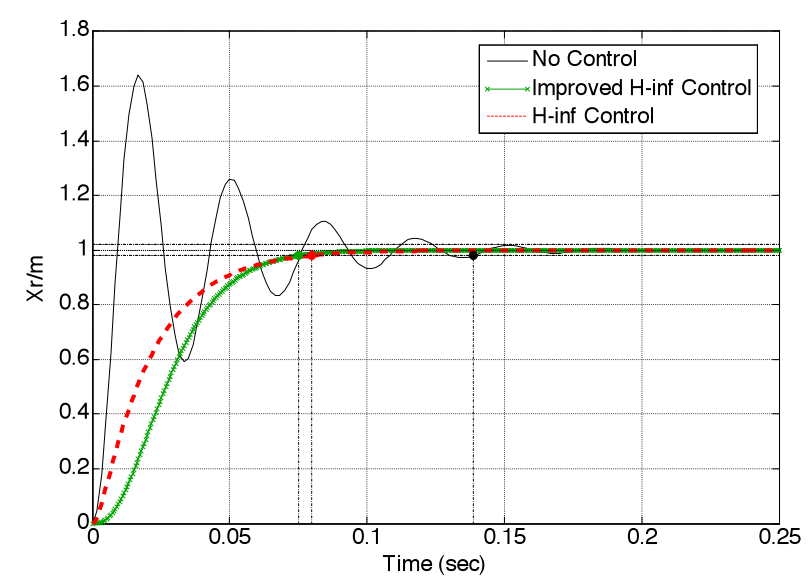

Figure 5. Step response curve of rack displacement

Analyzing the closed loop frequency spectrum of the controllers designed by the above two methods respectively and we can obtain the frequency spectrum as the figure 6 . According to figure, two different kinds controllers all can obtain the expected curve shape of $\mathrm{S} / \mathrm{T}$ curve; when the improved $\mathrm{H} \infty$ controller works, the gain of system sensitivity function is $0.316 \%$ in low frequency, the closed slope of closed loop is $-60 \mathrm{~dB} / \mathrm{dec}$; when the traditional $\mathrm{H} \infty$ controller works, the gain of system sensitivity function is $1.13 \%$ in low frequency, the closed slope of closed loop is $-40 \mathrm{~dB} / \mathrm{dec}$. So, the performance of robust and stability is not as perfect as the improved $\mathrm{H} \infty$ controller.

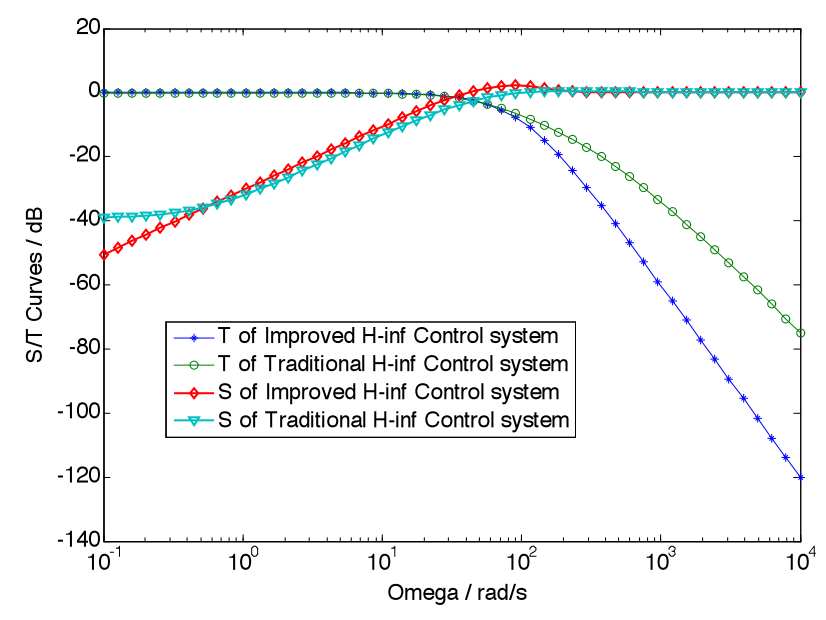

Figure 6. Frequency spectrum curve of SBW

\section{CONCLUSIONS}

This paper mainly discusses the dynamic modeling, analyzing and simplifying reasonably. Design the controller with the method based on the $\mathrm{H} \infty$ circuit shaping by deep analysis on the system. To avoid a large number of iterate calculus in selecting the weighting function of the classical $\mathrm{H} \infty$ arithmetic, put forward a new method to design an improved $\mathrm{H} \infty$ controller and design a controller for the SBW system. Simulate the system with the improved controller designed in this paper and traditional controller, then capture the results. The curves of closed loop frequency spectrum and the simulation results show that the method in this paper is simple and effective, and the improved controller has the better robust steady than the traditional controller. The interference step response shows that the new controller with the improved algorithm has a better robust performance. The results of the simulation taken under the condition of the parameter perturbation show that the new controller still can keep the system steady. These results declare that the controller design in this paper meet the control demands completely.

\section{REFERENCES}

[1] Haggag, S., Alstrom, D., Cetinkunt, S., Egelja, “A Modeling, control, and validation of an electro-hydraulic steer-by-wire system for articulated vehicle applications", IEEE /ASME Transactions on Mechatronics,No.6, 2005, pp.688-692.

[2] Neville A.Stanton,Phipip Marsden. "From fly-by-wire to driveby-wire:Safety implications of automation in Vehicles", Automotive engineering, Vol.109, No.9, 2001, pp.102-106.

[3] Choi S., Alonso J.J. and Kroo H.M. "Two-level multi-fidelity design optimization studies for supersonic jets", Journal of Aircraft, Vol.46, No.3, 2009, pp.776-790. http://dx.doi.org/ $10.2514 / 1.34362$

[4] Se-Wook OH, Ho-Chol CHAE, Seok-Chan YUN, et al. "The Design of a Controller for the Steer-by-Wire System", JSME international Journal Series C, Vol.47, No3, 2004, pp.38-44.

[5] M. Amrllein, P.T. Krein. "Dynamic simulation for analysis of hybrid electric vehicle systems and subsystems interactions, including power electronics", IEEE Trans. Veh. Technol. Vol. 54, No.3, 2005, pp. 825-836. http://dx.doi.org/10.1109/ TVT.2005.847231

[6] Xiang Chen, Tiebao Yang, Xiaoqun Chen, "A Generic ModelBased Advanced Control of Electric Power-Assisted Steering Systems", IEEE Transactions on Control Systems Technology. 
Vol. 16, 2008, pp. 1289-1300. http://dx.doi.org/10.1109/ TCST.2008.921805

[7] Paolo F., Francesco B., Jahan A., "Predictive active steering control for autonomous vehicle systems", IEEE Transactions on Control Systems Technology, Vol. 15, No. 3, 2007, pp. 566-580. http://dx.doi.org/10.1109/TCST.2007.894653

[8] Selamat H., Yusof R., Goodall R. M., "Self-tuning control for active steering of a railway vehicle with solid-axle wheel sets". IET Control Theory Appl., Vol. 5, No. 2, 2008, pp. 374-383. http://dx.doi.org/10.1049/iet-cta:20070111

\section{AUTHORS}

Chen Huipeng is with School of Mechanical Engineering, HangZhou DianZi University, Hangzhou , China (e-mail:hpchen@hdu.edu.cn).

Lingjun Zhu is with Vehicle Engineering Department, Hangzhou Dianzi University, Hangzhou, China(e-mail:lovezlj0603@163.com)
Liu Xiaofan is with Mechanical Engineering Department, Hangzhou Dianzi University, Hangzhou, China(e-mail:1xf090@126.com)

Yu Sunan is with Mechanical Engineering Department,HangZhou Dianzi University, Hangzhou, China(e-mail:540926979@qq.com).

Dingcheng Zhao is with Mechanical Engineering Department, Hangzhou Dianzi University, Hangzhou, China(e-mail:994148351@qq.com).

This work was supported in part by NSFC under Grant Nos. 51105125 . It is an extended and modified version of a paper presented at the International Conference on Mechanical Engineering, Automation and Material Science (MEAMS2012), held 22-23 December 2012, Wuhan, China. Received 14 February 2013. Published as resubmitted by the authors 25 March 2013. 